\title{
Las Tecnologías y la Enseñanza en la Educación Superior. Un Simulador Aplicado a la Integración de Conceptos Enseñados en Cursos de Posgrado
}

\section{Technology and Teaching in Higher Education. A Simulation Applied to the Integration of Concepts Taught in Postgraduate Courses}

\author{
Silvana L. Giudicessi*, María C. Martínez-Ceron*i, \\ Soledad L. Saavedra, Osvaldo Cascone y Silvia A. Camperi
}

Universidad de Buenos Aires ${ }^{\mathrm{ii}}$

\begin{abstract}
Introducción: El objetivo de este trabajo fue implementar tecnologías de la información y la comunicación (TIC) a nivel de posgrado. En este caso particular se usó USINA, un simulador para la toma de decisiones diseñado por el Centro de Innovaciones en Tecnología y Pedagogía (CITEP), de la Universidad de Buenos Aires. Ésta permite trabajar con narraciones, estudio de casos y utilizar el "error" como método pedagógico. Métodos: 1) Diseñar una secuencia de pantallas para ser montadas en USINA utilizándola como herramienta de integración de conceptos en dos cursos de posgrado: Downstream Processing de proteínas (DSP) y Aplicaciones, Síntesis y Análisis de Péptidos Sintéticos (ASAP). 2) Aplicar una encuesta anónima para evaluar la eficiencia de USINA. 3) Realizar una clase presencial para intercambiar opiniones sobre la experiencia. Resultados y Discusión: En ambos cursos (DSP y ASAP) la experiencia resultó exitosa. El alumnado consideró que el uso de USINA permitió el andamiaje de conocimientos y la integración de conceptos al situarlo en el rol de experto. El uso de las TIC en los niveles educativos superiores sigue siendo una asignatura pendiente. A través de la experiencia aquí analizada, se abre la posibilidad de llevar esta herramienta a cursos de pregrado y con mayor número de estudiantes.
\end{abstract}

Palabras clave: Simulador, Tecnología de la educación, Enseñanza superior, Integración, Biotecnología, Química, Tecnologías de la información y de la comunicación, Enseñanzaaprendizaje, Innovación pedagógica.

\begin{abstract}
Introduction: The aim of this work was to implement information and communication technologies (ICTs) at postgraduate level. In this particular case, USINA, a decision-making simulator designed by the Center for Innovation in Technology and Education (CITEP), of the University of Buenos Aires, was used. It allows working with stories, case studies and uses the "error" as a teaching method. Methods: 1) Design a sequence of screens to be mounted in the USINA platform to use it as a tool for the integration of concepts in two postgraduate courses: Protein Downstream Processing (DSP) and Applications, Synthesis and Analysis of Synthetic Peptides (ASAP). 2) Apply anonymous quiz to evaluate the efficiency of USINA. 3) Perform a class attendance to exchange opinions on the experience. Results: During both courses (DSP and ASAP) the experience was very successful. The students felt that the use of USINA enhanced learning and helped integrating concepts by situating them in the role of experts. Discussion: The use of ICTs in higher levels of education remains a pending issue. Through the experience here analyzed the possibility to bring this tool to undergraduate courses and more students is opened.
\end{abstract}

Keywords: Simulation, Educational technology, Higher education, Integration, Biotechnology, Chemistry, Information and communication technologies, Teachinglearning, Educational innovation.

*Contacto:sgiudicessi@ffyb.uba.ar camartinez@ffyb.uba.ar

issn: 1989-0397

www.rinace.net/riee/

https://revistas.uam.es/riee
Recibido: $\quad 8$ de marzo de 2016

$1^{\text {a }}$ Evaluación: 24 de abril de 2016

Aceptado: 15 de mayo de 2016 


\section{Introducción}

La incorporación de nuevas herramientas a la enseñanza en el nivel superior es un desafío que merece especial atención. La utilización de viejos métodos y la persistencia de metodologías de enseñanza dificultan la innovación en la educación superior. En un escenario de sobreabundancia de información, la principal preocupación de los docentes sigue siendo formar a los alumnos para el futuro, para que se desempeñen exitosamente como profesionales. El tradicional modo de enseñanza, donde el docente simplemente transmite conocimientos a sus alumnos, se presenta como una posible propuesta, pero no única, e incluso en algunos casos, insuficiente. Reconocer el aprendizaje como un proceso de interacción social abre la oportunidad al desarrollo de nuevas estrategias de enseñanza de las disciplinas y a la manipulación de nuevas aplicaciones en constante renovación (Jacubovich, 2012).

\section{Fundamentación teórica}

\subsection{Uso de TIC en la enseñanza de nivel superior}

Con el uso de las computadoras han aparecido nuevas formas de aprendizaje para la enseñanza de las ciencias básicas que posibilitan su acercamiento a alumnos. Las tecnologías de la información (TIC) aparecen como recursos didácticos a través de entornos virtuales tales como el campus virtual, los laboratorios virtuales y los simuladores que brindan la posibilidad de trabajar en un ambiente de enseñanza e investigación de tipo "protegido", con prácticas de muy bajo costo a las que no se tendría acceso de otro modo y que además se pueden reproducir las veces que fueran necesarias hasta apropiarse de los conceptos (Cabero, 2007).

El uso de las TIC en la enseñanza ha tenido una profunda repercusión en los últimos años, ya que ha demostrado ser una herramienta de gran valor para mejorar la enseñanza por parte de los docentes y el aprendizaje por parte de los alumnos.

En 2008 se realizó un estudio sobre el uso de las tecnologías en la Argentina en el cual se encontró que la telefonía móvil se había cuadruplicado en los últimos años, los usuarios de Internet en el país llegaban a duplicar el promedio mundial y existían 3,7 millones de conexiones a Internet, de las cuales el 90\% eran de banda ancha (Bernal, González, Ojeda y Zanfrillo, 2011). Este masivo consumo de la tecnología en la vida cotidiana también refleja un aumento de su utilización en la educación. Como expresa Cardelli (2007), las TIC han permitido incorporar nuevas fuentes de información y aumentar la rapidez para adquirirlas. Además, la reforma curricular en las universidades, junto con la implementación de las TIC y nuevas políticas que las incluyen y fomentan, permitió el aumento considerable de métodos que involucran tecnologías informáticas y de comunicación (López, 2007). La utilización de la tecnología y la informática en la educación (al igual que en la vida cotidiana) se está desarrollando actualmente a nivel mundial. Por ejemplo, todos los países europeos cuentan con estrategias nacionales para fomentar el uso de las TIC en diferentes ámbitos, incluyendo una estrategia específica dedicada a la educación. En muchos casos estas estrategias están encaminadas a proporcionar a los alumnos competencias en el manejo de las TIC (en particular alfabetización digital), así como a proporcionar formación en TIC a los docentes (EACEA, 2011). En todos los países los grupos destinatarios de estas medidas son los 
profesores/formadores, en tanto que las actividades se centran sobre todo en las etapas de educación primaria y secundaria. Sin embargo, de a poco se está implementando también en la educación superior. De esta manera, diferentes herramientas tecnológicas van adquiriendo protagonismo en las universidades e institutos de educación superior. Uno de los elementos tecnológicos más usados es el campus virtual, el cual Del Bello (2001) define como "un medio de comunicación y un medio de almacenamiento de información”. El campus ha tenido una enorme repercusión en la educación superior, ya sea por su gran utilidad como por su amplia aplicación para la enseñanza y el aprendizaje. En la Universidad de Buenos Aires, desde hace casi una década, se ha venido implementando el uso del campus virtual suministrado por Moodle, como principal medio de comunicación alternativo, como archivo virtual, bibliográfico y guía de trabajos prácticos, y para inscripción a materias, entre otros. Su diseño simple y didáctico lo ha convertido en una herramienta utilizada en varias universidades, como es el caso de la Universidad Nacional de Quilmes, que cuenta desde hace varios años con la Universidad Virtual (UVQ), la cual se encuentra sostenida por la plataforma virtual y año tras año incorpora mayor cantidad de usuarios. Desde entonces, no solamente se han registrado mayores demandas en el uso de las fuentes tecnológicas en la Universidad de Quilmes; en otras universidades, como es el caso de la Universidad Nacional de La Plata, el funcionamiento de muchos ámbitos de docencia, extensión e investigación utiliza TIC en forma cada vez más intensiva. Se han implementado políticas que promueven el mantenimiento y mejoramiento de las redes que permiten el acceso y el manejo de los entornos virtuales y herramientas de la red, lo cual confirma la importancia que la tecnología cobra en la educación en todos los niveles en nuestro tiempo (Díaz, Molinari y Raimundo, 2007).

A día de hoy, y como ya resaltaba Carnoy en 2004, no se han desarrollado ampliamente nuevas realidades con modelos pedagógicos basados en las TIC de acuerdo a lo que la demanda universitaria exige. Si bien las políticas ya han sido implementadas, las propias TIC aplicadas en las aulas e incluso en la vida cotidiana de los propios alumnos no han tenido una gran aceptación aún. La utilización de los entornos virtuales como una de las herramientas de las TIC ha colaborado con lo que hoy conocemos como la educación a distancia, en complementariedad con la presencial, lo que permite una mejor calidad educativa y más equitativa para aquellos que no pueden concurrir a clases. Para Salinas (2004), lograr entornos virtuales de enseñanza-aprendizaje efectivos, es decir, que permitan obtener buenos resultados en términos de construcción de conocimientos, requiere considerar cambios metodológicos y en las estrategias didácticas que allí se despliegan. En una investigación realizada por Tumino y Bournissen (2014) se advierte la tendencia a establecer patrones de uso de técnicas y herramientas de aprendizaje tecnológicas, conforme a la intención de provocar la creatividad y el descubrimiento en el ejercicio formativo. Sin embargo, la escasa participación docente renuente a la implementación de estas nuevas tecnologías es todavía la gran limitación que no permite potenciar las estrategias de aprendizaje de los estudiantes mediante el uso de las TIC. Tecnologías de comunicación e información ofrecidas, como las plataformas virtuales tipo Moodle (que presentan, entre otras cosas, los portafolios, los WebQuest, los foros y los aportes colaborativos de las Wiki), son utilidades que no han sido aún suficientemente exploradas y estratégicamente explotadas en el área de la enseñanza (Tumino y Bournissen, 2014). Desde el Centro de Innovaciones en Tecnología y Pedagogía (CITEP, http://citep.rec.uba.ar), perteneciente al Rectorado de la 
Universidad de Buenos Aires, se ofrecen cursos con el objeto de fomentar y generar espacios pedagógicos, creando un gran potencial para implementar el uso de nuevas herramientas didácticas.

Para aprovechar todo el potencial de las TIC habría que utilizarlas en el aula no solo como herramientas para el aprendizaje, sino también para la evaluación.

Hoy día, en los cursos impartidos, generalmente se hace uso del foro de discusión y chat, ya que es importante combinar el uso de las nuevas tecnologías con los métodos tradicionales de enseñanza. Esto tiene por objeto satisfacer las demandas de cambio y adaptaciones permanentes detectadas en las necesidades actuales del alumnado y brindar al estudiante experiencias potencialmente transferibles a otras situaciones que involucren el manejo de estrategias y métodos de trabajo. La interacción a través del foro, con los docentes y los propios pares, permite a los estudiantes un andamiaje constante, lo cual potencia sus aprendizajes. El uso de las TIC debe integrarse a un cambio de paradigma basado en el aprendizaje, haciendo, compartiendo y colaborando en contextos que propicien la innovación y creatividad. En este sentido se recupera la importancia del auto-aprendizaje y las habilidades para encontrar información confiable, desarrollar ideas innovadoras, comunicar información y evaluar las soluciones creativas (Cataldi, Lage y Dominghini, 2013).

Otro modelo de enseñanza en entornos virtuales es el uso de simuladores, que propone a los alumnos construir conocimiento acerca de complejas relaciones entre variables en el marco de un contexto que reproduce la complejidad en la que estas variables se manifiestan y sobre las que los alumnos deben intervenir (Pinto, 2006).

\subsection{Simulaciones para la enseñanza universitaria}

Cataldi et al. (2013) definen los simuladores como programas que buscan reproducir situaciones de la vida real mediante la visualización de los diferentes estados que el mismo puede presentar, donde cada estado está definido y descripto por un conjunto de variables que cambian mediante la interacción en el tiempo con un algoritmo determinado, a fin de describir de manera intuitiva el comportamiento del sistema real, dado que operar sobre este es inaccesible. Permite diseñar un modelo del sistema real y realizar experimentos con este modelo, a fin de comprender el comportamiento del sistema y evaluar las distintas estrategias operativas del sistema en estudio.

La tecnología ha proporcionado las herramientas y métodos para que en el ambiente de simulación puedan convivir videos, animaciones, gráficos interactivos, audio y narraciones (Casanovas, 2005).

La importancia de las simulaciones reside en hacer partícipe al usuario de una vivencia para desarrollar hábitos, destrezas y esquemas mentales que influyan en su conducta. A su vez, permite la resolución de situaciones problemáticas, a través de la reflexión y el razonamiento (Arroyo, 2014). La simulación es una de las herramientas más poderosas disponibles para los responsables en la toma de decisiones, tales como ingenieros, diseñadores, analistas, administradores y directivos, para diseñar y operar sistemas complejos. Ejemplo de esto son los simuladores de vuelo y de conducción de automóviles, entre otros. Muchos de estos simuladores están disponibles en Internet y abiertos al público general, aunque la mayoría se encuentra en inglés (Bio Network, 2004; Gel Electrophoresis, 2009; Virtual Lab, 2008). 
Como señala Arroyo (2014) una cuestión relevante en el uso de simuladores en educación es la disminución de la posibilidad de cometer errores durante la práctica profesional. Esto es de suma importancia en carreras propias del área de la salud, tal como en el caso de Medicina y Psicología (Huamaní, 2014), ya que disminuyen la posibilidad de generar un perjuicio relevante en los pacientes. Así como en carreras administrativas los riesgos se reducen a la pérdida de un cliente (Osorio, Ángel y Franco, 2012), en el área de la Biotecnología estos pueden ser los mismos que en las carreras comerciales y en el área de la Salud, donde la importancia radica en los efectos sobre un paciente.

A la hora de enseñar ciencias "duras" los estudiantes pueden encontrarse con un entorno educativo no amigable, con un proceso de enseñanza caracterizado por memorizar hechos, seguir protocolos como si fueran recetas de cocina y escuchar de forma pasiva en clase (Bonde et al., 2014). Labster (https://www.labster.com/) ofrece entornos virtuales online para la generación de simuladores aplicados a la enseñanza, ya que son una fuente de estímulos sensoriales y cognitivos que permiten que los estudiantes pongan en juego sus ideas frente a las interacciones que plantea el desarrollo de la actividad realizada en el simulador. Bonde et al. (2014) analizaron uno de los diez simuladores que se encuentran en Labster: un simulador de un laboratorio de criminalística. Tomaron 91 estudiantes de un curso introductorio de Ciencias Biológicas. Al comienzo del curso se les realizó un examen de nivel. Luego dividieron el curso en dos, a una mitad se les dio una clase tradicional con exposición del docente y a la otra mitad se les pidió que recorrieran un simulador. Luego, a todo el alumnado se lo evaluó con un examen. Invirtieron los grupos para la siguiente lección y fueron nuevamente evaluados. Tras un período de tiempo, se les pidió a los alumnos que repitieran los exámenes para evaluar la capacidad de retención. Con estos resultados concluyeron que el uso de este simulador incrementó ampliamente la capacidad de integrar los conocimientos, aunque esto no influyó en la aprobación de los exámenes parciales.

Salas y Ardanza (1995) propusieron el uso de simuladores para la enseñanza de las Ciencias Médicas. Ellos analizaron exhaustivamente las ventajas y desventajas del uso de simulaciones mediadas por computadora y concluyeron la importancia que tiene especialmente en el ciclo clínico-epidemiológico de las carreras de Ciencias Médicas, ya que permite realizar una práctica semejante a la que sucede en la práctica profesional.

Para Gokhale (1991), las estrategias de aprendizaje basadas en el desarrollo de habilidades de pensamiento de orden superior implican tres principios: a) la creación de un ambiente cautivador para el que aprende, b) la combinación de experiencias de aprendizaje visuales e interactivas que ayuden a los estudiantes a formar representaciones mentales y c) el desarrollo de la arquitectura cognitiva que integre las experiencias de aprendizaje. Las simulaciones interactivas de computadora basadas en estas estrategias ayudan a los estudiantes a crear las explicaciones sobre los sucesos, a discutir y argumentar la validez de esas explicaciones, tendiendo puentes entre los estilos de aprendizaje de los estudiantes y los estilos de enseñanza de los docentes.

Desde la perspectiva docente, el diseño de un simulador significa realizar un complejo replanteo pedagógico, desde la estrategia didáctica hasta la configuración del entorno virtual (Arroyo, 2014). Se deben diseñar escenarios compatibles con la práctica profesional, en los que se pongan en juego no solo los conocimientos teóricos adquiridos durante el proceso de enseñanza/aprendizaje, sino que permitan modificar la posición 
del alumno a través del lenguaje (Katz, 1996), ya que en lugar de usar indicaciones como recitar o definir, propios de un nivel 1 de la clasificación taxonómica de Bloom, se utilizan otras propias del nivel 6, en el que principal comando será "decidir". Esto les permitiría no sólo realizar tareas de manera automática sino llevarlos al análisis, a la evaluación y a la propuesta de diferentes puntos de vista para la resolución del problema (o juego de problemas) al que se enfrenten los alumnos.

Aldrich (2005) propone una clasificación de los tipos de simuladores. USINA, según esta clasificación, pertenecería al tipo de "historia ramificada", en la cual el alumnado se apodera de la situación del proceso de enseñanza, ya que cada vez que se le presenta una posibilidad de tomar una decisión, esta conlleva a una consecuencia. Así, los guía a involucrarse de manera activa con su propio proceso de aprendizaje, utilizando los conocimientos adquiridos previamente y permitiendo la integración de los mismos.

\subsection{USINA aplicado al proceso de enseñanza/aprendizaje}

Los simuladores, como USINA (http://usina.rec.uba.ar), propician el aprendizaje estimulando la búsqueda de estrategias superadoras y la toma de decisiones similares a las que ocurren en el campo profesional. Es un simulador en español, diseñado por el CITEP, orientado a la enseñanza y aprendizaje en el nivel superior. La construcción de la situación-problema inicial, como también de las alternativas a elegir y los nuevos escenarios que cada elección genera, conforman la clave de la experiencia. Se trata de una experiencia pedagógica diseñada para investigar, analizar y tomar decisiones con el objeto de integrar los aprendizajes académicos y promover habilidades ligadas a la práctica o el ejercicio profesional desde una mirada disciplinar o interdisciplinar (Jacubovich, 2012).

USINA promueve que el docente piense en algún problema de enseñanza relevante, que genere un árbol rico en ramificaciones y derivaciones (Lion, Soletic, Jacubovich y Gladkoff, 2011), y se basa en dos tipos de aprendizaje: el aprendizaje en base a la resolución de casos y el aprendizaje en base a la resolución de problemas.

El docente diseña una propuesta de enseñanza mediada tecnológicamente, por lo que USINA se convierte en una "herramienta de autor" para cada una de las propuestas. No se trata solamente de pensar en respuestas correctas o incorrectas, sino en vías posibles de resolución que conllevarán distintas consecuencias. En todos los casos, en el momento final de la simulación se ofrece una devolución docente del camino recorrido.

La estructura básica de USINA está conformada por:

a) Un contexto: marco espacial y temporal y las características del entorno en el cual se desarrollará el problema.

b) Un escenario: en el cual se brinda la información acerca del rol que el alumnado va a asumir dentro del problema.

c) Un primer problema: donde se presenta una situación inicial o tarea concreta que el alumnado tiene que resolver para comenzar a dar una solución al caso.

d) Alternativas posibles de solución al primer problema planteado.

e) Materiales e información de los que se puede valer el alumnado para la toma de decisiones.

f) Toma de decisión entre las alternativas propuestas que conlleva una consecuencia concreta. 
g) Siguientes problemas con sus posibles soluciones en los cuales el alumnado irá recorriendo diferentes caminos en base a las decisiones tomadas y donde cada problema subsiguiente es consecuencia de la decisión tomada en el problema anterior.

h) El resultado o final del recorrido al que arribará el alumnado según las decisiones que haya ido tomando en las diferentes etapas. El mismo se acompaña con una devolución del docente.

Se presentan, a su vez, dos pantallas. En una de ellas se muestra el "historial de decisiones", el cual consiste, una vez alcanzado uno de los posibles resultados, en la presentación de las decisiones tomadas a través de la simulación. Y en la otra, el "recorrido óptimo", que muestra la secuencia de decisiones correctas.

En el presente trabajo se planteó el uso de la USINA en cursos de posgrado de pocos alumnos (entre 12 y 15 alumnos). Estos cursos son intensivos y en dos semanas se imparten gran cantidad de conocimientos. Son adecuados para alumnos que deben viajar y faltar a sus trabajos para su formación. Es por ello un gran desafío poder integrar todos los temas tratados en tan poco tiempo. En nuestra Cátedra (Cátedra de Biotecnología, Facultad de Farmacia y Bioquímica, Universidad de Buenos Aires), durante 2015 se dictaron dos cursos de posgrado. El primero, llamado Downstream Processing de Proteínas (DSP), tiene como objetivo enseñar a elegir procesos de recuperación y purificación de proteínas obtenidas por medio de procesos biotecnológicos. Se busca diseñar el proceso más eficiente y de menor costo para que sea factible la fabricación industrial del producto biotecnológico. El segundo curso, llamado Aplicaciones, Síntesis y Análisis de Péptidos Sintéticos (ASAP), enseña diferentes metodologías de obtención de péptidos sintéticos y sus diferentes aplicaciones en la industria.

Dado que ambas áreas de conocimiento presentan dificultades a la hora de realizar el proceso de enseñanza/aprendizaje y en la integración de los conocimientos adquiridos, se consideró el uso de simuladores como herramienta de integración de dichos cursos.

A su vez, se seleccionaron estos cursos por contar con pocos alumnos, lo cual facilitaría la realización de una prueba piloto con el objeto de evaluar su posible aplicación en las materias de grado que se dictan en la Cátedra. En ambos cursos se invitaba al alumnado a recorrer la simulación en la segunda etapa de la cursada, en la cual ya contaban con los conocimientos necesarios para realizarla. En ambos casos, el alumnado se ponía en el rol del experto que era convocado por una empresa. En el caso de DSP, se necesitaba la purificación de una proteína y, en el caso de ASAP, la síntesis de una biblioteca de péptidos que sería utilizada para la purificación de una proteína. En el escenario, se informaba al estudiante del rol que debía tomar y se presentaba el problema a resolver. La pantalla siguiente conducía al alumnado a la primera decisión a tomar, con una o más opciones. Si el alumnado elegía la opción correcta, se le permitía continuar con la USINA hacia la siguiente toma de decisiones; en caso contrario, se lo despedía o, simplemente, no era seleccionado por la empresa, y se lo invitaba a recorrer nuevamente la USINA para que realizara un nuevo recorrido. 


\section{Método}

El uso de simuladores para la enseñanza en posgrado no tiene antecedentes en la Cátedra de Biotecnología, donde se realizaron los cursos antes citados. No es el caso del uso de TICs ya que la Cátedra cuenta con un Campus virtual desde hace más de 5 años.

El curso de DSP lleva siendo impartido desde hace más de 10 años. Antes de encarar el diseño del simulador, se tenía conocimiento de ciertas dificultades a la hora de integrar los conceptos dictados durante el curso en tan corto período de tiempo, más aún si se considera que el alumnado es muy heterogéneo como suele suceder en cursos de posgrado.

Conociendo estas dificultades epistemológicas, fue una preocupación de los docentes encontrar una herramienta que pudiera permitir superarlas. Se tomó entonces contacto con los profesionales del CITEP para diseñar por medio de USINA una simulación que fuera un apoyo didáctico para dicho curso. Se decidió usar un simulador en parte por las ventajas previamente nombradas, pero también porque el alumnado que realiza cursos de posgrado en su mayoría son nativos digitales (Huamaní, 2012). Los entornos virtuales de enseñanza y aprendizaje tienden a estimular el aprender haciendo, utilizando un medio con el que el nativo digital se encuentra familiarizado.

En el momento que se pensó el curso de ASAP, la experiencia obtenida con el curso de DSP fue inspiradora. Dado el resultado positivo no solo observado por el alumnado sino también por el equipo docente, se pensó en el simulador como herramienta central a la hora de integrar los conocimientos dictados durante este curso. El equipo docente tiene una amplia experiencia en enseñar las metodologías para la síntesis química de péptidos $y$, aunque esta fue la primera vez que se dictó como curso, ha capacitado varias generaciones de tesistas doctorales, lo que hacía que se estuviera familiarizado con las dificultades que el temario podía presentar al alumnado.

Se seleccionó un simulador del tipo "historia ramificada" porque no sólo se buscaba la integración de los conocimientos adquiridos a lo largo del curso sino también, estimular el aprendizaje colaborativo y estimular la toma de decisiones, actividades imprescindibles que realiza cualquier profesional. Considerando que el alumnado está compuesto por adultos cursando estudios de posgrado, esta metodología permite poner en juego una práctica lúdica apropiada para que puedan sentirse involucrados con las responsabilidades propias del campo profesional.

Los cursos elegidos para la implementación de USINA tienen un alto contenido teórico y práctico. Son cursos de 60 horas cada uno, con una duración de dos semanas. Al ser intensivos, los alumnos reciben una gran cantidad de información y conceptos en un período corto de tiempo, lo que hace difícil el correcto andamiaje del conocimiento.

El Diseño Educativo consta de varios pasos a seguir para la elaboración de un proceso de diseño y desarrollo de la propuesta educativa. Estos pasos serían la elaboración de los contenidos del cual el equipo docente es el responsable. La información se estructura según una situación real y concreta que puede ser fácilmente entendida por el alumnado. Los contenidos se estructuran en un formato educativo virtual en función del aprendizaje significativo, que en el caso de USINA son situaciones reales en las que se debe tomar una decisión, y cada toma de decisiones conlleva a una nueva pantalla con nuevas decisiones a tomar. Este simulador permite insertar los recursos necesarios para 
optimizar la construcción de los conocimientos en forma significativa. Así se concreta el diseño de la propuesta educativa. Posteriormente, se realizan las etapas de seguimiento y monitoreo de las actividades ejecutadas por el alumnado. Finalmente, como propone Bautista (2015), a partir de los informes extraídos de la aplicación, los docentes a cargo del curso realizarán un informe final de la retroalimentación grupal e individual, tanto de la propuesta como del ejercicio de los simuladores, al utilizar los foros y la puesta en común en el nivel presencial.

La propuesta didáctica fue diseñada como diagramas de flujo para luego ser montados en el entorno USINA, en el cual se plantearon una serie de pantallas.

En el planteo del simulador del curso DSP se diseñaron tres tipos de pantallas: "Contexto", "Escenario" y "Pantalla problema" (figura 1):

$\checkmark$ "Contexto": Se describen las características de la empresa donde se suscita el caso problemático, se describe dónde está situada y quiénes son los posibles competidores.

$\checkmark$ "Escenario": Se sitúa al alumno en el rol de especialista en DSP de proteínas y al cual se le solicita que diseñe un proceso de purificación de una proteína de aplicación industrial (insulina), la cual se encuentra en una mezcla compleja.

$\checkmark$ Pantalla problema 1: Se presenta el problema con la primera toma de decisiones, en el cual se le pide que decida qué tipo de métodos analíticos utilizaría para la caracterización de la muestra entre una batería de métodos a elección. Entre esta batería, sólo algunos métodos le serán de utilidad para la caracterización necesaria de la muestra compleja y se propicia que el alumnado evalúe la metodología analítica más adecuada a su problemática.

A partir de esta pantalla, se suceden las diferentes pantallas propias del árbol de decisión diseñado para que vaya obteniendo información sobre la proteína a purificar y sus contaminantes y nuevos problemas que lleven a una purificación exitosa, en el caso de que se seleccionen las opciones correctas. Si el alumnado selecciona las opciones incorrectas o menos óptimas, la siguiente pantalla tendrá una explicación sobre cuál fue el inconveniente que presenta la/s opción/es elegida/s. A su vez, se dejaron preguntas que serían contestadas de forma presencial, con la intervención del grupo y del docente.

De la misma manera que en en el caso del curso de DSP, se plantearon tres tipos de pantallas para el curso de ASAP: "Contexto", "Escenario" y "Pantalla problema" (figura 2):

$\checkmark$ "Contexto": Se describen las características del lugar de trabajo donde se suscita el caso problemático, se describe dónde está situada y quiénes son los posibles competidores.

$\checkmark$ "Escenario": Se sitúa al alumnado en el rol de especialista en síntesis de péptidos en fase sólida. En este escenario se le explica que se quiere sintetizar una biblioteca peptídica de determinadas características y utilizarla para purificar un anticuerpo.

$\checkmark$ Pantalla problema 1: Se presenta la primera toma de decisiones.

Según el proceso que el alumnado seleccione, será conducido a una pantalla con las explicaciones referentes a las ventajas y desventajas de las decisiones que tome, de acuerdo al camino elegido en la simulación. Si el alumnado selecciona las opciones 
incorrectas o menos óptimas, la siguiente pantalla tendrá una explicación sobre cuál fue el inconveniente que presenta la/s opción/es elegida/s.

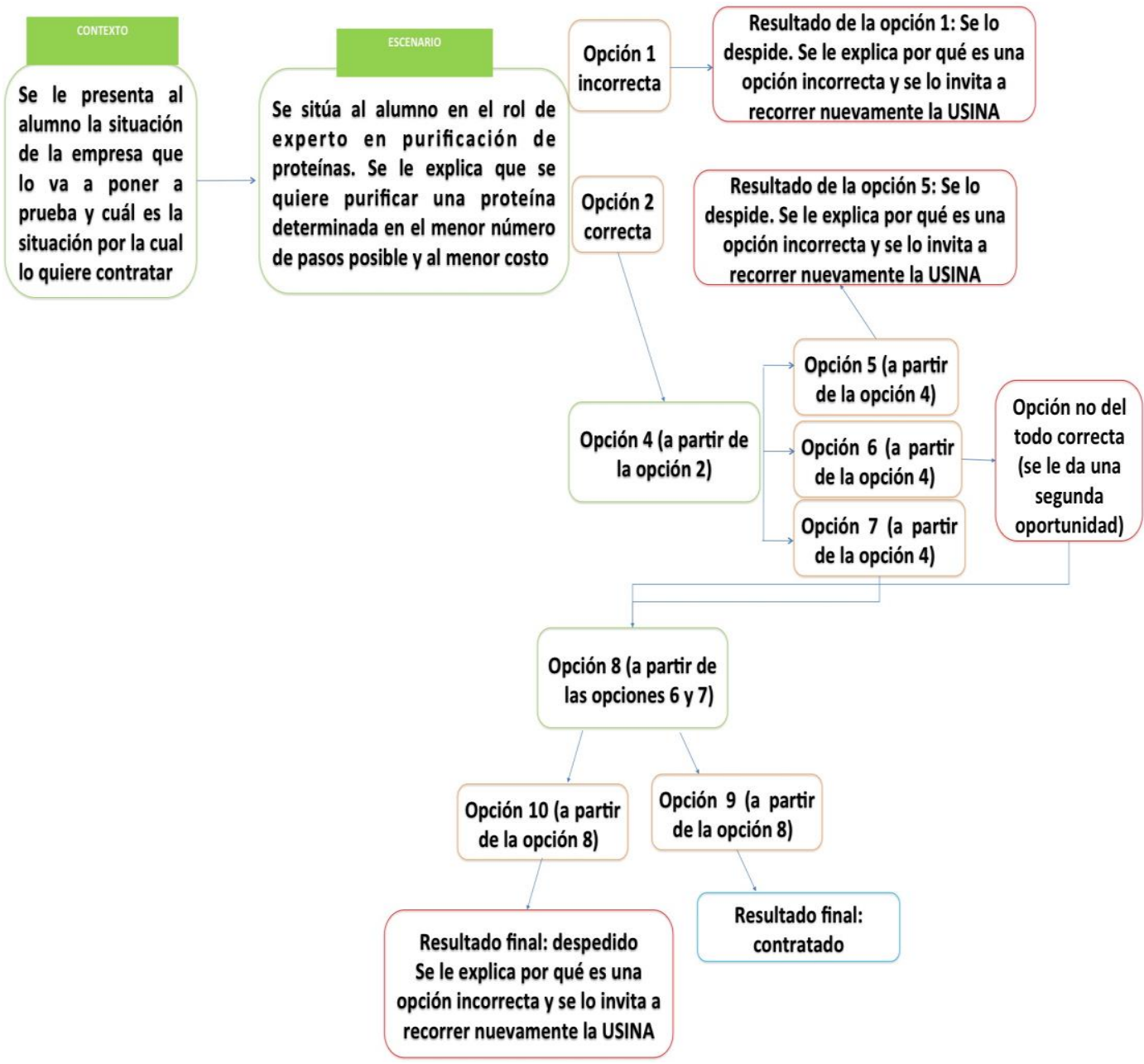

Figura 1. Esquema de un problema tipo de Downstream Processing de proteínas planteado en USINA (DSP)

Fuente: Elaboración propia.

Finalmente, se programó una instancia presencial y participativa para hacer una puesta en común de los recorridos realizados y contestar las preguntas que se planteaban al final de los recorridos.

Para evaluar la eficacia de USINA como herramienta pedagógica se diseñó y entregó a los alumnos, tanto del curso de DSP como de ASAP, una encuesta anónima (Tabla 1). 


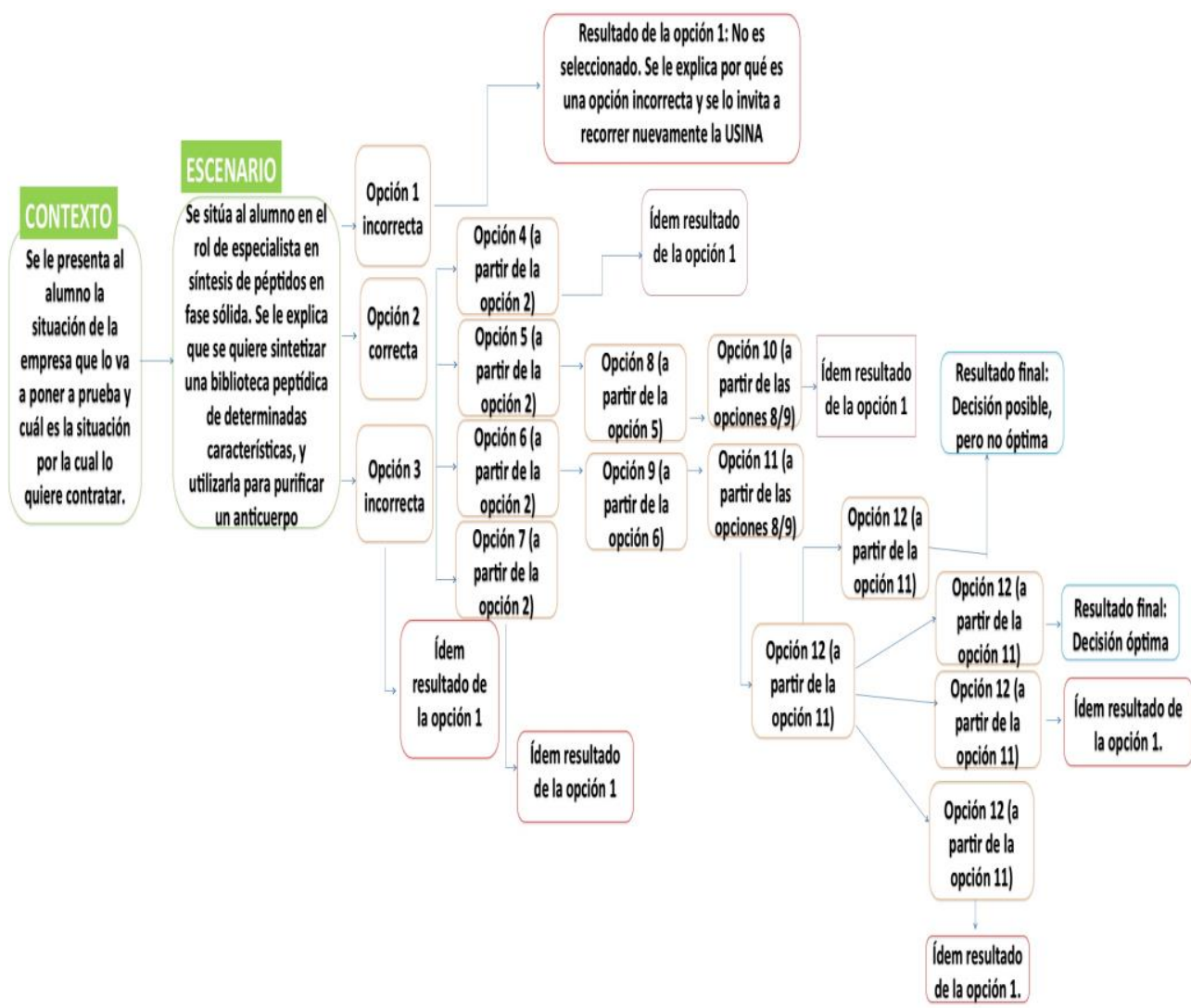

Figura 2. Esquema de un problema tipo planteado en USINA para el curso de Aplicaciones, Síntesis y Análisis de Péptidos Sintéticos (ASAP)

Fuente: Elaboración propia.

Tabla 1. Encuesta para evaluar la eficiencia de USINA entregada a los alumnos de los cursos DSP y ASAP

1- Sexo del alumno

2- Edad del alumno

3- ¿Cuál es su título de grado?

4- Especifique la institución otorgante. En el caso que haya respondido "Otra" en la pregunta anterior, aclarar cuál, por favor.

5- ¿Los conocimientos brindados en el curso fueron adecuados para resolver los problemas planteados en USINA?

6- ¿La bibliografía otorgada durante la cursada y la presente en USINA fueron suficientes?

7- ¿El tiempo para resolver los problemas de USINA fue suficiente?

8- ${ }_{i}$ El uso del simulador no presentó ninguna dificultad?

9- ¿El camino elegido hasta la respuesta obtenida, haya sido o no la "óptima", permitió evaluar lo aprendido y entender los resultados obtenidos?

10- ¿El uso de USINA, permitió integrar, repasar y evaluar los conceptos aprendidos durante el curso?

11- El uso de Twitter, foro, apoyo docente resultaron útiles (esta pregunta no aparece en el cuestionario de ASAP)?

12- ¿El uso de la USINA promovió la participación de los alumnos?

13- ¿Considera que el uso de USINA marcó una diferencia entre antes y después de su uso?

14- ¿Por qué?

15- ¿Recomendaría el uso de USINA para otras materias?

16- Por favor, detalle por qué

17- ¿Qué cambios sugiere en la aplicación de USINA?

Fuente: Elaboración propia. 


\section{Resultados}

\subsection{Estadísticas de USINA}

El programa USINA permite llevar un registro de los intentos y las diferentes decisiones tomadas por cada estudiante. El docente puede ver el camino que cada uno realizó y así verificar si la decisión tomada fue correcta o incorrecta. Cuando se diseñó la USINA para el curso de DSP, la meta final era ser "contratado", lo cual sucedía en la medida que las decisiones tomadas a lo largo del recorrido fueran mayoritariamente correctas. En el caso de la USINA de ASAP el estudiante era seleccionado como becario de un grupo de investigación.

Del total que realizaron el curso de DSP, el 50\% fueron contratados en el primer intento. El 83\% de la segunda mitad fueron contratados en un segundo intento.

En el curso de ASAP, el 50\% fue seleccionado en un primer intento. Mientras que de la otra mitad, el $50 \%$ fue seleccionado en un segundo intento.

\subsection{Resultados de las encuestas}

A continuación se muestran los resultados de las encuestas tanto para el curso de DSP como el de ASAP.

\subsubsection{Análisis de la implementación de USINA en el curso de DSP}

En el caso del curso de DSP, el $67 \%$ de los estudiantes era de sexo femenino y más del $66 \%$ presentaba una edad menor a 30 años (Figuras $3 \mathrm{~A}$ y $3 \mathrm{~B}$ ). El $11 \%$ del alumnado pertenecía a la carrera de Bioquímica, el $22 \%$ a la carrera de Biotecnología, el $11 \%$ a la carrera de Farmacia, el $11 \%$ a la de Química, el 33\% a Veterinaria y el $11 \%$ a Microbiología (Figura 3C). Del total, el 66,67\% provenían de universidades nacionales estatales, el $11,11 \%$ de universidades privadas y el $22,22 \%$ de universidades extranjeras (Figura 3D).

Respecto de la información suministrada en la Figura 4, el 100\% de los encuestados opinaron que los contenidos de la materia fueron los adecuados para resolver la USINA, ya sea en mayor o menor medida (pregunta 5), mientras que el $89 \%$ del alumnado coincidió en que la bibliografía otorgada en la cursada fue suficiente para resolver el problema planteado (pregunta 6). En la mayoría de los casos, el tiempo otorgado para realizar la simulación fue suficiente (pregunta 7); sin embargo, es necesario considerar que se trataba de un curso de posgrado cuya duración era de 2 semanas. En el caso de tratarse de una materia cuatrimestral de grado, el tiempo hubiese sido mayor ya que el alumnado podría comenzar a transitar la USINA una o dos semanas antes de finalizar la cursada. En cuanto a las dificultades encontradas en el uso del simulador, se observaron problemas relacionados con la plataforma online pero no con el diseño de la USINA en sí. Producto de ello, se elaboró una nota al CITEP solicitando mejorar el sistema para que fuera más sencillo habilitar a los alumnos en el uso del simulador (pregunta 8). La mayoría del alumnado afianzó los conocimientos mediante el uso del simulador, independientemente del recorrido realizado y de haber llegado a una de las respuestas óptimas. El uso del simulador les permitió repasar e integrar lo aprendido durante el curso, e incluso los caminos que conducían a la respuesta "incorrecta" también permitieron el andamiaje de conocimientos incorporados durante el curso. Cabe destacar que los cursos de posgrado que se dictan en la Cátedra suelen ser intensivos para facilitar su cursada por alumnos de otras regiones del país y del extranjero. En poco 
tiempo se imparten gran cantidad de conocimientos, lo que atenta contra la integración y real comprensión de los contenidos. El uso de USINA permitió integrar los conocimientos de una manera más completa y simple, al mismo tiempo que promovió el aprendizaje en cada toma de decisión, sea correcta o incorrecta (preguntas 9 y 10). Como complemento, y para promover la aplicación de otras herramientas de las TICs, se ofreció un espacio de difusión, discusión y consulta en un foro y una cuenta de Twitter. Estas herramientas no fueron tan útiles como se esperaba (pregunta 11), principalmente debido a que los alumnos asistían a clases diariamente y el trato con los docentes era personalizado. Estas herramientas pueden ser muy útiles en materias cuatrimestrales, con grupos numerosos y divididos en turnos. En estos casos, estas herramientas que permiten un trato alumno-docente más cercano y personal pueden ser de gran utilidad para realizar consultas, solicitar información bibliográfica e incluso enriquecer los conocimientos a través de debates entre los propios alumnos.

La participación del alumnado se vio positivamente incrementada en la clase presencial de debate con la implementación de USINA, ya que fue necesaria la discusión y puesta en común de los conocimientos y conceptos adquiridos para analizar la experiencia personal en el uso de la plataforma. El alumnado se involucró seriamente, al tomar la posición de "expertos" en el tema, y participó activamente de las consultas de otros compañeros (pregunta 12).

Estos resultados marcan un antes y un después en el uso de USINA (pregunta 13), ya que los estudiantes no solo reconocieron una mejor comprensión gracias al uso de la simulación, sino que consideran recomendable su uso en otros cursos.

Si bien las preguntas 14 a la 17 fueron de opinión, de ellas se desprende que cuando se le pidió al alumnado que valorara esta nueva herramienta, las justificaciones se referían a la integración de conceptos enseñados y la importancia de lo aprendido al involucrarse en un rol profesional dentro de una simulación de trabajo real y la posibilidad de una autoevaluación (pregunta 14). Esto llevó a que todos los alumnos recomendaran la plataforma para otras materias (preguntas 15 y 16). En la pregunta 17, la mayoría proponía mejoras en la plataforma pero asociadas a los inconvenientes técnicos que habían experimentado. 


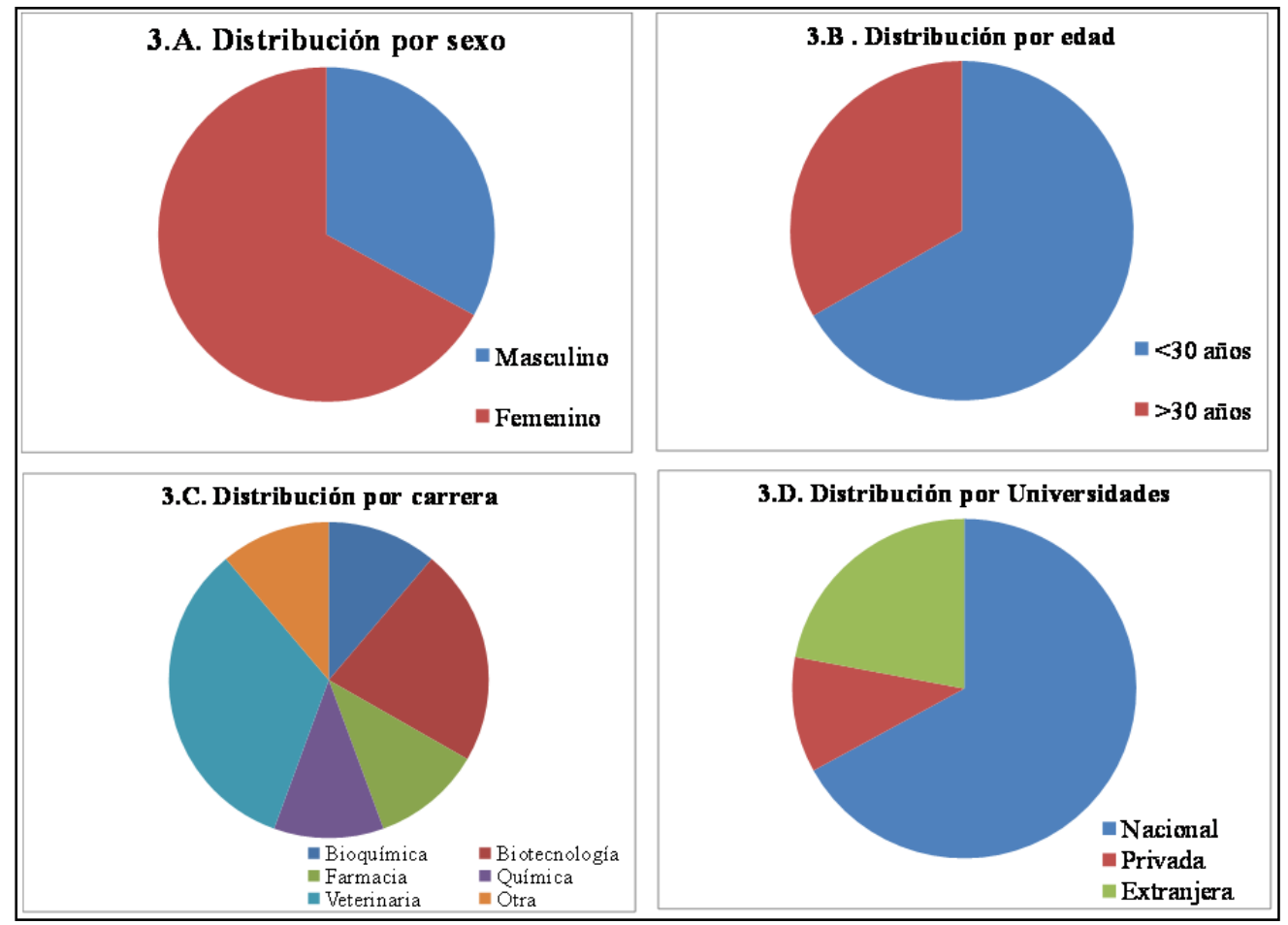

Figura 3. Resultados de la encuesta sobre USINA realizada durante el curso de Downstream Processing de Proteínas (DSP): A) distribución por sexo, B) distribución por edad, C) distribución por carrera y D) distribución por universidad Fuente: Elaboración propia.

\subsubsection{Análisis de la implementación de USINA en el curso de ASAP}

En este curso, el $71 \%$ del alumnado era de sexo femenino y menor de 30 años (Figuras $5 \mathrm{~A}$ y $5 \mathrm{~B}$ ). El 14\% (Figura 5C) había realizado la carrera de Biología, el 43\% de Biotecnología y otro 43\% pertenecían a otras carreras (Genética, Ingeniería en Alimentos). Todos habían hecho su carrera en universidades estatales nacionales, a diferencia de los alumnos que realizaron el curso de DSP, que presentaba mayor heterogeneidad.

En la información suministrada en la Figura 6 se puede observar que todos los encuestados coincidieron en que la USINA pudo ser resuelta con la información suministrada en el curso, en tiempo y forma (preguntas 5-7). En cuanto a las dificultades para realizar la simulación, estas se refirieron principalmente a problemas técnicos propios de la plataforma, por lo que los alumnos tuvieron que esperar para poder acceder a la misma (pregunta 8). La mayor parte del grupo pudo integrar y afianzar los conocimientos mediante la USINA, independientemente de las decisiones tomadas y de alcanzar o no resultados óptimos en la resolución del problema. 


\section{USINA en el curso de DSP}

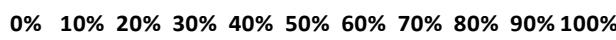

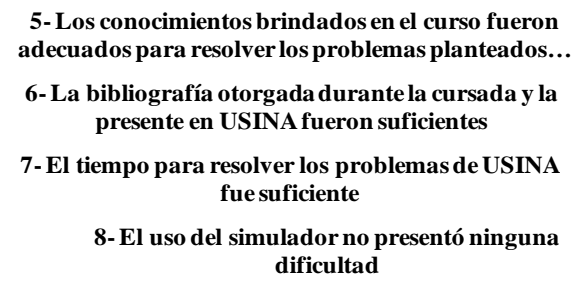

5- Los conocimientos brindados en el curso fueron adecuados para resolver los problemas planteados... 6- La bibliografía otorgada durante la cursada y la presente en USINA fueron suficientes

7-EI tiempo para resolver los problemas de USINA fue suficiente

8-El uso del simulador no presentó ninguna dificultad

9- El camino elegido hasta la respuesta obtenida, haya sido o no la "óptima”, permitió.

10-El uso de USINA, permitió integrar, repasar y evaluar los conceptos aprendido durante el curso

11-El uso de Twitter, foro, apoyo docente resultaron útiles

12- El uso de la USINA promovió la participación de los alumnos

13- ¿Considera que el uso de USINA marcó una diferencia entre antes y después de su uso?

Estoy totalmente de acuerdo $\quad$ Estoy de acuerdo Estoy parcialmente de acuerdo

Figura 4. Resultados de la encuesta sobre USINA realizada durante el curso de Downstream Processing de Proteínas (DSP): Distribución de respuestas de la 5 a la 13 Fuente: Elaboración propia.

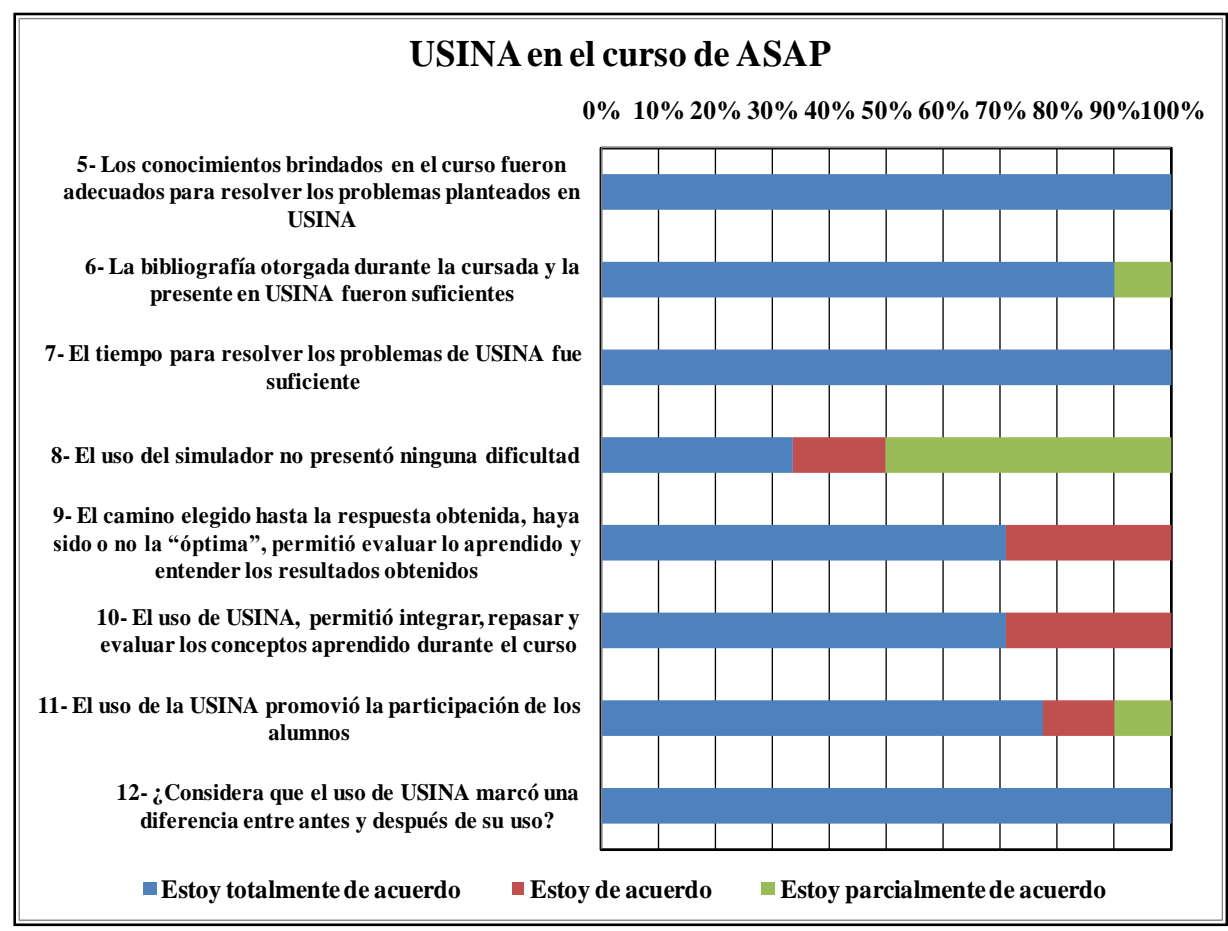

Figura 5. Resultados de la encuesta sobre USINA realizada durante el curso de Aplicaciones, Síntesis y Análisis de Péptidos Sintéticos (ASAP): A) distribución por sexo, B) distribución por edad y C) distribución por carrera Fuente: Elaboración propia. 
La estrategia ayudó a afianzar los conocimientos adquiridos en el curso. Nuevamente, y como se observó en el curso de DSP, el 100\% del alumnado recomendó el uso de USINA en otras materias y, además, los alumnos expresaron que la misma los había ayudado en la integración de los conocimientos, la autoevaluación y para realizar un repaso y síntesis de lo aprendido.

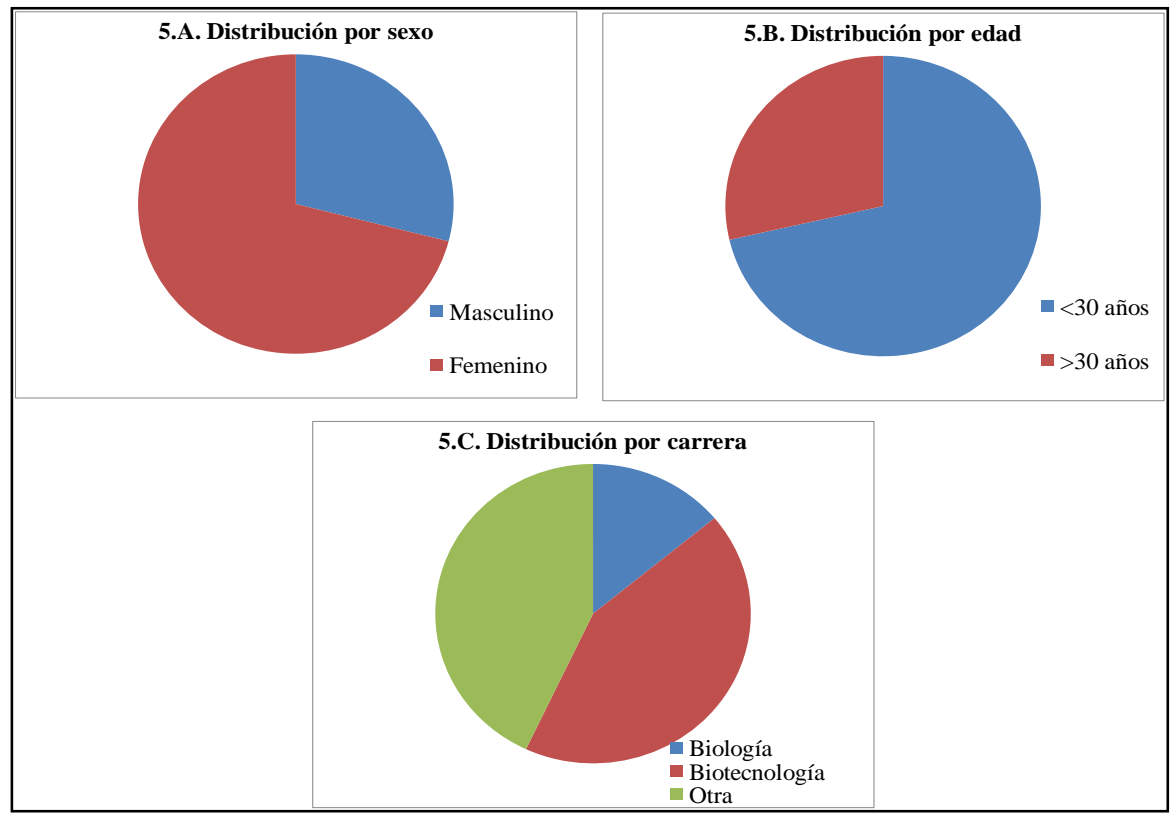

Figura 6. Resultados de la encuesta sobre USINA realizada durante el curso de Aplicaciones, Síntesis y Análisis de Péptidos Sintéticos (ASAP): Distribución de respuestas de la 5 a la 13 Fuente: Elaboración propia.

\section{Discusión}

USINA ofrece una alternativa para trabajar con el "Error", tal como lo propone Astolfi (1999), permitiendo transparentar las decisiones que realiza el alumnado en diferentes resultados que son consecuencia de las decisiones por ellos tomadas a lo largo de un recorrido que tiene como fin la resolución de un caso semejante al que se les puede presentar en el ejercicio de su profesión.

Los alumnos no solo aceptaron la propuesta pedagógica sino que cuando se les pidió que dieran su opinión consideraron que para mejorar la USINA se debían incorporar más preguntas y temas de debate, para integrar aún más todos los temas vistos en cada curso. Esto muestra que la USINA es un sistema más ameno, simple y didáctico que el alumnado prefiere a la hora de repasar y autoevaluar los conocimientos adquiridos. Esta plataforma fue una elección preferencial para los alumnos en su experiencia de aprendizaje, y no solo valoraron su utilidad en el curso sino también como una potencial herramienta para otras materias.

A pesar de lo propuesto por Cataldi et al. (2013) sobre el uso de foros, nosotros observamos que no resultó útil en este caso, posiblemente por el tipo de curso dictado, pero no descartamos que para cursos más numerosos pueda ser una herramienta imprescindible. 
Cabe destacar la estrecha colaboración que surgió con el CITEP, que no solo acompañó el diseño de las simulaciones sino que respondió prontamente para solucionar los inconvenientes que fueron surgiendo con la plataforma online. El uso de la plataforma USINA está abierto para toda la Universidad de Buenos Aires. Para otras universidades existe la posibilidad de usarlo bajo convenio.

Es nuestro objetivo a futuro incrementar la complejidad y la interacción entre los estudiantes y la plataforma para acrecentar la sensación de realismo. Para ello, creemos que se debe buscar mayor interacción con actores de otras disciplinas, tales como licenciados en informática, pedagogos y biotecnólogos, entre otros.

Finalmente, creemos que la implementación de USINA para cursos de posgrado y de poca cantidad de alumnos resulta prometedora para seguir con el paso posterior de ensayar su uso en materias más numerosas y de pregrado, lo cual será implementado próximamente.

\section{Referencias}

Aldrich, C. (2005). The four traditional simulation genres. En R. Taff. (Ed.), Learning by doing: The essential guide to simulations, computer games, and pedagogy in e-learning and other educational experiences (pp. 3-6). Nueva York: John Wiley \& Sons.

Arroyo Fernández, C. (2014). Diseño pedagógico del simulador SIPAD. Recurso para la formación investigadora inicial del profesorado de Educación Primaria y la atención a la diversidad en el aula (Tesis de grado, Universidad de Sevilla, Sevilla).

Astolfi, J. P. (1999). El "error", un medio para enseñar. Sevilla: Díada.

Bautista Pinzón, M. A. (2015). Evaluación de herramientas virtuales utilizadas para la formación de psicólogos y la adquisición de competencias específicas para la profesión: Estudio preliminar. Organización de los Estados Americanos: Virtual Educa: Foros Virtuales. Recuperado de http://reposital.cuaed.unam.mx:8080/jspui/handle/123456789/4511

Bernal Escoto, B., González Carella, M. I., Ojeda Orta, M. E. y Zanfrillo, A. I. (2011). Brecha digital en la transferencia de conocimientos: Educación superior en Argentina y México. Florianópolis: INPEAU.

Bio Network. (2004). Carolina del Norte, EEUU: North Carolina Community College System. Recuperado de http://www.ncbionetwork.org/

Bonde, M. T., Makransky, G., Wandall, J., Larsen, M. V., Morsing, M., Jarmer, H. y Sommer, M. O. A. (2014). Improving biotech education through gamified laboratory simulations. Nature Biotechnology, 32(7), 694-697. doi:10.1038/nbt.2955

Cabero Almenara, J. (2007). Las TICs en la enseñanza de la química: Aportaciones desde la tecnología educativa. En A. Bodalo (Ed.), Química: Vida y progreso (pp. 1-36). Murcia: Asociación de Químicos de Murcia.

Cardelli, J. (2007). Educación superior, transnacionalización y virtualización. Un estudio de caso en la Argentina. En F. López Segrega (Ed.), Escenarios mundiales de la educación superior. Análisis global y estudios de casos (pp. 283-304). Buenos Aires: CLACSO.

Carnoy, M. (2004). Las TIC en la enseñanza: Posibilidades y retos. Lección inaugural del curso académico 2004-2005 de la UOC. Recuperado de http://www.uoc.edu/inauguralo4/esp/carnoy 1004.pdf 
Casanovas I. (2005). La didáctica en el diseño de simuladores digitales para la formación universitaria en la toma de decisiones (Tesis para Magister en Docencia Universitaria, Universidad Tecnológica Nacional, Facultad Regional de Buenos Aires, Buenos Aires).

Cataldi, Z., Lage, F. J. y Dominghini, C. (2013). Fundamentos para el uso de simulaciones en la enseñanza. Revista de Informática Educativa y Medios Audiovisuales, 10(17), 8-16.

Del Bello, J. C. (2001). Educación por Internet en Argentina: El caso de la Universidad Nacional de Quilmes. Revista Iberoamericana de Ciencia, Tecnología, Sociedad e Innovación, 1.

Díaz, J., Molinari, L. y Raimundo, M. (2007). Usos y políticas de las TICs en la Educación Superior en la Argentina: El caso de la Universidad Nacional de La Plata (UNLP). Recuperado de http://www.utn.edu.ar/aprobedutec07/docs/192.pdf

EACEA (Agencia Ejecutiva en el Ámbito Educativo, Audiovisual y Cultural, Comisión Europea). (2011). Cifras claves sobre el uso de las TIC para el aprendizaje y la innovación en los centros escolares de Europa. Bruselas: Eurydice.

Eisner, E. W. (1994). Cognición y currículum: Una visión nueva. Buenos Aires: Amorrortu.

Gel Electrophoresis. (2009). Utah, Estados Unidos: University of Utah. Recuperado de http://learn.genetics.utah.edu/content/labs/gel/

Gokhale, A. A. (1991). Effectiveness of computer simulation versus lab and sequencing of instruction, in teaching logic circuits. Journal of Industrial Teacher Education, 29(1), 1-12.

Huamaní, C. G. A. (2014). Simulation and virtual learning environments: Tools for teaching psychology in higher education. Psychology Research, 4(5), 376-382.

Jacubovich, J. (2012). La enseñanza con simuladores en Educación Superior. El caso de Usina. En M. J. Alfano, S. Mantegazza, G. Sarmiento y V. Scabone (Eds.), III Jornadas Nacionales y I Latinoamericanas de Investigadores/as en Formación en Educación Instituto de Investigaciones en Ciencias de la Educación (IICE) (pp. 69-81). Buenos Aires: Facultad de Filosofía y Letras, Universidad de Buenos Aires. Recuperado de http://iice.institutos.filo.uba.ar/sites/iice.institutos.filo.uba.ar/files/14Tecnolog\%C3\%ADa_O.pdf

Katz, M. (1996). Teaching organic chemistry via student-directed learning: A technique that promotes independence and responsibility in the student. Journal of Chemical Education, 73(5), 440-441. doi:10.1021/edo73p440

LABSTER. (2016). Dinamarca: Labster. Recuperado de https://www.labster.com/

Lion, C., Soletic, A., Jacubovich, J. y Gladkoff, L. (2011). Las tecnologías y la enseñanza en la educación superior. El caso de usina como herramienta de autor. Revista Iberoamericana de Evaluación Educativa, 4(2), 107-117.

López Segrera, F. (2007). Notas para un estudio comparado de la educación superior a nivel mundial. En F. López Segrega (Ed.), Escenarios mundiales de la educación superior. Análisis global y estudios de casos (pp. 21-104). Buenos Aires: CLACSO.

Osborne, J. y Hennessy, S. (2003). Literature review of ICT: Promise, problems and future directions. Bristol: Futurelab.

Osorio Villa, P. A., Ángel Franco, M. B. y Franco Jaramillo, A. (2012). El uso de simuladores educativos para el desarrollo de competencias en la formación universitaria de pregrado. Revista Q 7(13), 1-23.

Pinto, L. (2006). Tecnología e innovación pedagógica en el Nivel Superior. Asesoría Pedagógica, Facultad de Farmacia y Bioquímica. Buenos Aires: Facultad de Farmacia y Bioquímica, Universidad de Buenos Aires: 
Salas Perea, R. S. y Ardanza Zulueta, P. (1995). La simulación como método de enseñanza y aprendizaje. Revista Cubana de Educación Médica Superior, 9, 1-2.

Salinas Ibáñez, J. (2004). Cambios metodológicos con las TIC. Estrategias didácticas y entornos virtuales de enseñanza-aprendizaje. Bordón, 56(3-4), 476-480.

Tumino, M. C. y Bournissen, J. M. (2014). Herramientas informáticas: Uso en procesos de enseñanza-aprendizaje de entornos virtuales. European Scientific Journal, 1O(13), 27-46.

VIRTUAL LAB. (2008). USDA Cooperative State Research, Education, and Extension Service \& USDA Estados Unidos: National Institute of Food and Agriculture. Recuperado de http://virtuallabs.nmsu.edu/index.php

\section{Breve CV de los autores}

\section{Silvana L. Giudicessi}

Participa en el dictado de materias de grado de las carreras de: Farmacia, Bioquímica y Licenciatura en Ciencias y Tecnología de los Alimentos de la Universidad de Buenos Aires (UBA) y de numerosos cursos de posgrado. Realizó la Carrera Docente en la Facultad de Farmacia y Bioquímica (FFyB) y cursos de capacitación docente orientados en el uso de las TICs en educación. Es investigadora del CONICET y como resultado de su desempeño académico es autora de 13 artículos con referato, 3 capítulos de libros, una patente de invención y 36 presentaciones a reuniones científicas. En el año 2014 recibió un premio al Mejor Trabajo por el trabajo titulado "USINA aplicada a la enseñanza del Downstream processing de proteínas de interés industrial”. Ha recibido 3 premios. Ha participado en la creación de 3 plataformas USINAS para mejorar la enseñanza en Biotecnología y Química Orgánica.

\section{María C. Martínez-Ceron}

Participa en el dictado de materias de grado de las carreras de: Farmacia, Bioquímica y Licenciatura en Ciencias y Tecnología de los Alimentos de la Universidad de Buenos Aires (UBA), como profesora de Farmacia de la Universidad Argentina J. F. Kennedy y de numerosos cursos de posgrado. Realizó la Carrera Docente en la FFyB y cursos de capacitación docente orientadas al uso de TICs en educación. Es investigadora del CONICET y como resultado de su desempeño académico es autora de 14 artículos con referato, 4 capítulos de libros, dos patentes de invención y 30 presentaciones a reuniones científicas. Participó como coautora en el trabajo presentado y premiado en SAPROBIO 2014 antes nombrado. Ha recibido 6 premios. Es co-creadora de 3 plataformas USINA para mejorar la enseñanza en Biotecnología y Química Orgánica.

\section{Soledad L. Saavedra}

Participa en el dictado de materias de grado de las carreras de: Farmacia, Bioquímica y Licenciatura en Ciencias y Tecnología de los Alimentos de la Universidad de Buenos Aires (UBA) y de numerosos cursos de posgrado. Participó como coautora en el trabajo presentado y premiado en SAPROBIO 2014 antes nombrado. Ha recibido 6 premios. Es co-creadora de 3 plataformas USINA para mejorar la enseñanza en Biotecnología y Química Orgánica. A su vez está realizando su tesis doctoral en el área Biotecnología en 
la Cátedra de Biotecnología de la FFyB de la UBA. En el 2007 recibió el título de Licenciada en Biotecnología con orientación en Genética Molecular. Como parte de su desempeño académico posee un trabajo publicado y uno en prensa, dos capítulos de libros, cuatro presentaciones a reuniones científicas y ha realizado numerosos cursos.

\section{Osvaldo Cascone}

Profesor Consulto de la FFyB, Académico Titular de la Academia Nacional de Farmacia y Bioquímica, Investigador Superior del CONICET. Es un referente en el área de Expresión y purificación de proteínas y Biotecnología. Ha dirigido 17 tesis doctorales, 4 de Maestría en Biotecnología. Ha dictado 65 cursos de posgrado de la especialidad purificación de proteínas. Ha publicado 134 artículos en revistas con referato, 11 capítulos de libros y 9 patentes de invención. Ha participado en 267 reuniones científicas nacionales e internacionales. Ha participado en numerosos proyectos de investigación nacionales e internacionales. En el 2015 recibió el premio a la trayectoria docente universitaria otorgado por el Rectorado de la Universidad de Buenos Aires. Participó como coautora en el trabajo presentado y premiado en SAPROBIO 2014 antes nombrado. Ha recibido numerosos premios. Es co-creador de 1 plataforma USINA para mejorar la enseñanza en Biotecnología.

\section{Silvia A. Camperi}

Profesora Adjunta en la Cátedra de Biotecnología de la FFyB de la UBA e Investigadora Independiente del CONICET. Participa en el dictado de materias de grado de las carreras de: Farmacia, Bioquímica y Licenciatura en Ciencias y Tecnología de los Alimentos de la UBA y de numerosos cursos de posgrado. Recibió en 1997 el título de Docente con Formación Pedagógica en Enseñanza Universitaria y en el 2006 el de Docente Autorizada de la UBA. Participa en el dictado de materias de grado y posgrado en la FFyB. Ha dirigido 2 tesis doctorales y dirige actualmente a 2 Investigadores del CONICET y 3 Becarios de Doctorado. Ha participado en más de 20 proyectos de investigación. Publicado 9 capítulos de libros, 35 en revistas científicas con referato y 5 patentes de invención. Ha recibido numerosos premios. Participó como coautora en el trabajo presentado y premiado en SAPROBIO 2014 antes nombrado. Es co-creadora de 3 plataformas USINA para mejorar la enseñanza en Biotecnología y Química Orgánica.

\footnotetext{
' Silvana L. Giudicessi y María C. Martínez-Ceron participaron de forma equivalente en el artículo.

ii Cátedra de Biotecnología, Facultad de Farmacia y Bioquímica, Universidad de Buenos Aires. Junín 956, 6to piso (1113), Ciudad Autónoma de Buenos Aires, Argentina.
} 